\title{
A tight quantitative version of Arrow's impossibility theorem
}

Received March 21, 2010

\begin{abstract}
The well-known Impossibility Theorem of Arrow asserts that any generalized social welfare function (GSWF) with at least three alternatives, which satisfies Independence of Irrelevant Alternatives (IIA) and Unanimity and is not a dictatorship, is necessarily non-transitive. In 2002, Kalai asked whether one can obtain the following quantitative version of the theorem: For any $\epsilon>0$, there exists $\delta=\delta(\epsilon)$ such that if a GSWF on three alternatives satisfies the IIA condition and its probability of non-transitive outcome is at most $\delta$, then the GSWF is at most $\epsilon$ away from being a dictatorship or from breaching the Unanimity condition. In 2009, Mossel proved such a quantitative version, with $\delta(\epsilon)=\exp \left(-C / \epsilon^{21}\right)$, and generalized it to GSWFs with $k$ alternatives, for all $k \geq 3$.

In this paper we show that the quantitative version holds with $\delta(\epsilon)=C \epsilon^{3}$, and that this result is tight up to logarithmic factors. Furthermore, our result (like Mossel's) generalizes to GSWFs with $k$ alternatives. Our proof is based on the works of Kalai and Mossel, but uses also an additional ingredient: a combination of the Bonami-Beckner hypercontractive inequality with a reverse hypercontractive inequality due to Borell, applied to find simultaneously upper bounds and lower bounds on the "noise correlation" between Boolean functions on the discrete cube.
\end{abstract}

\section{Introduction}

Consider an election procedure in which a society of $n$ members selects a ranking amongst $k$ alternatives. In the voting process, each member of the society gives a ranking of the alternatives (the ranking is a full linear ordering; that is, indifference between alternatives is not allowed). The set of rankings given by the individual members is called a profile. Given the profile, the ranking of the society is determined according to some function, called a generalized social welfare function (GSWF).

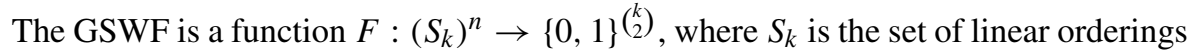
on $k$ elements. In other words, given the profile consisting of linear orderings supplied by the voters, the function determines the preference of the society amongst each of the $\left(\begin{array}{l}k \\ 2\end{array}\right)$ pairs of alternatives. If the output of $F$ can be represented as a full linear ordering of the $k$ alternatives, then $F$ is called a social welfare function (SWF).

Throughout this paper we consider GSWFs satisfying the Independence of Irrelevant Alternatives (IIA) condition: For any pair of alternatives $A$ and $B$, the preference of the

N. Keller: Department of Mathematics, Bar Ilan University, Ramat Gan 52900, Israel; e-mail: nkeller@math.biu.ac.il 
entire society between $A$ and $B$ depends only on the preference of each individual voter between $A$ and $B$. This natural condition on GSWFs can be traced back to Condorcet [6].

Condorcet's paradox demonstrates that if the number of alternatives is at least three and the GSWF is based on the majority rule amongst every pair of alternatives, then there exist profiles for which the voting procedure cannot yield a full order relation. That is, there exist alternatives $A, B$, and $C$ such that the majority of the society prefers $A$ over $B$, the majority prefers $B$ over $C$, and the majority prefers $C$ over $A$. Such a situation is called non-transitive outcome of the election.

In his well-known Impossibility Theorem [1], Arrow showed that this paradox occurs for any "reasonable" GSWF satisfying the IIA condition:

Theorem 1.1 (Arrow). Consider a generalized social welfare function $F$ with at least three alternatives. If the following conditions are satisfied:

- The IIA condition,

- Unanimity: if all the members of the society prefer some alternative A over another alternative $B$, then $A$ is preferred over $B$ in the outcome of $F$,

- $F$ is not a dictatorship (that is, the preference of the society is not determined by a single member),

then the probability of a non-transitive outcome is positive (i.e., there necessarily exists a profile for which the outcome is non-transitive).

Since the existence of profiles leading to a non-transitive outcome has significant implications on voting procedures, an extensive research has been conducted in order to evaluate the probability of non-transitive outcome for various GSWFs. Most of the results in this area are summarized in [9]. In addition to its significance in social choice theory, this area of research leads to interesting questions in probabilistic and extremal combinatorics (see [20]).

In 2002, Kalai [14] suggested an analytic approach to this study. He showed that for GSWFs on three alternatives satisfying the IIA condition, the probability of a nontransitive outcome with respect to a uniform distribution of the individual preferences can be computed by a formula related to the Fourier-Walsh expansion of the GSWF. Using this formula he presented a new proof of Arrow's Impossibility Theorem under the additional assumption of neutrality (i.e., invariance of the GSWF under permutation of the alternatives), and established upper bounds on the probability of non-transitive outcome for specific classes of GSWFs.

While providing an analytic proof to Arrow's theorem does not seem such an important goal (as there are several simple proofs of it, see [10]), Kalai aimed at establishing a quantitative version of the theorem. Such a version would show that for any $\epsilon>0$, there exists $\delta=\delta(\epsilon)$ such that if a GSWF on three alternatives satisfies the IIA condition and its probability of non-transitive outcome is at most $\delta$, then the GSWF is at most $\epsilon$ away from being a dictatorship or from breaching the Unanimity condition. Kalai indeed proved such a statement for neutral GSWFs on three alternatives, with $\delta(\epsilon)=C \epsilon$ for a universal constant $C$. 
Kalai [15] asked whether his techniques can be extended to general GSWFs, and suggested using the Bonami-Beckner hypercontractive inequality $[4,3]$ in order to get such an extension. However, Keller [17] showed by an example that a direct extension cannot hold: if there exists $\delta(\epsilon)$ as above, then it cannot depend linearly on $\epsilon$. Keller asked whether for general GSWFs on three alternatives, a quantitative version holds with $\delta(\epsilon)=C \epsilon^{2}$.

Mossel [18] succeeded in proving a quantitative version of Arrow's theorem for general GSWFs on three alternatives. Furthermore, he generalized his result to GSWFs on more than three alternatives, and to more general probability distributions on the individual preferences. Unlike Kalai's techniques, Mossel's proof is quite complex. While Kalai's proof uses only simple analytic tools but no combinatorial tools, Mossel's proof extends and exploits a combinatorial proof of Arrow's theorem given by Barbera [2]. Furthermore, it uses heavier analytic tools, including a reverse hypercontractive inequality of Borell [5] and a non-linear invariance principle introduced by Mossel et al. [20]. The only drawback in Mossel's result is the dependence of $\delta$ on $\epsilon: \delta(\epsilon)=\exp \left(-C / \epsilon^{21}\right)$ for a universal constant $C$, which seems far from being optimal. Mossel conjectured that the correct dependence of $\delta$ on $\epsilon$ should be polynomial. ${ }^{1}$

In this paper we present a tight quantitative version of Arrow's theorem for general GSWFs. We show that the dependence of $\delta$ on $\epsilon$ is indeed polynomial, and compute the exact dependence, up to logarithmic factors.

Before we present our results, we should specify the notion of "the distance of a GSWF on $k$ alternatives satisfying the IIA condition from a dictatorship or from breaching the Unanimity condition". We consider two different definitions of this notion. In both, the underlying probability measure is the uniform measure on $\left(S_{k}\right)^{n}$ (the set of all possible profiles).

The first definition measures the distance of the GSWF under examination from the family of GSWFs on $k$ alternatives which satisfy the IIA condition and whose output is always transitive. This family was partially characterized by Wilson [22], and fully characterized by Mossel [18]. It essentially consists of combinations of dictatorships with constant functions (see Section 2.3 for the exact characterization).

Definition 1.2. Denote by $\mathcal{F}_{k}(n)$ the family of GSWFs on $k$ alternatives which satisfy the IIA condition and whose output is always transitive. For a GSWF $F$ on $k$ alternatives that satisfies the IIA condition, let

$$
D_{1}(F)=\min _{G \in \mathcal{F}_{k}(n)} \operatorname{Pr}[F \neq G] .
$$

We note that this is the definition that was used in [18]. Our main result for this definition is the following:

\footnotetext{
1 We note that Mossel also obtained another variant of his theorem, in which the dependence of $\delta$ on $\epsilon$ is $\delta(\epsilon)=C \epsilon^{3} n^{-3}$, where $n$ is the number of voters, and $C$ is a "decent" constant. As follows from our results presented below, this variant is essentially tight for very small values of $\epsilon$ (dependent on $n$ ). Moreover, for certain choices of parameters (specifically, "relatively small" $n$ and $\epsilon$ very small as a function of $n$ ), this result gives a stronger bound than our result, due to the better value of the constant.
} 
Theorem 1.3. There exists an absolute constant $C$ such that for any $k$ and for any GSWF $F$ on $k$ alternatives that satisfies the IIA condition, if the probability of non-transitive outcome in $F$ is at most

$$
\delta(\epsilon)=C\left(\epsilon / k^{2}\right)^{3},
$$

then $D_{1}(F) \leq \epsilon$.

For the second definition, we note that a GSWF $F$ on $k$ alternatives that satisfies the IIA condition actually consists of $\left(\begin{array}{l}k \\ 2\end{array}\right)$ independent Boolean functions $F_{i j}$ that represent the choice functions amongst the pairs of alternatives $(i, j)$ (for $1 \leq i<j \leq k$ ). The second definition is given in terms of these functions.

Definition 1.4. Denote by $\mathcal{G}_{2}(n)$ the set of constant functions and dictatorships on two alternatives. For a GSWF $F$ on $k$ alternatives that satisfies the IIA condition, let

$$
D_{2}(F)=\min _{1 \leq i<j \leq k} \min _{G \in \mathcal{G}_{2}(n)} \operatorname{Pr}\left[F_{i j} \neq G\right],
$$

where $\left\{F_{i j}\right\}_{1 \leq i<j \leq k}$ are as defined above.

Our main result for this definition is the following:

Theorem 1.5. There exists an absolute constant $C$ such that for any $k$ and for any GSWF $F$ on $k$ alternatives that satisfies the IIA condition, if the probability of non-transitive outcome in $F$ is at most

$$
\delta(\epsilon)=C \epsilon^{\frac{9\left(\sqrt{\log _{2}(1 / \epsilon)}+1 / 3\right)^{2}}{8 \log _{2}(1 / \epsilon)}},
$$

then $D_{2}(F) \leq \epsilon$.

Note that for small values of $\epsilon$, the exponent of $\epsilon$ in (1) tends to $9 / 8$.

We show that the dependence of $\delta$ on $\epsilon$ in Theorems 1.3 and 1.5 is tight, up to logarithmic factors in $\epsilon$. The examples showing the tightness are GSWFs on three alternatives, in which all the three choice functions $F_{12}, F_{23}, F_{13}$ are monotone threshold functions. In the example of Theorem 1.3, the expectations of the choice functions are $0,1-\epsilon, 1-\epsilon$ (in particular, one of the functions is constant!), and in the example of Theorem 1.5, the expectations are $\epsilon, 1 / 2,1-\epsilon$.

As in the works of Kalai and Mossel, the techniques we use are mainly analytic. Our proof essentially consists of three steps:

1. We consider a GSWF $F$ on three alternatives, and use a modification of Kalai's formula to express the probability of non-transitive outcome as a linear combination of "noise correlations" between the Boolean functions $F_{12}, F_{23}, F_{13}$ (see Section 2.2 for the definition of noise correlation).

2. We show that if at least one of the functions $F_{12}, F_{23}, F_{13}$ is close enough to a constant function, then the Bonami-Beckner hypercontractive inequality $[4,3]$ and a reverse hypercontractive inequality due to Borell [5] can be applied to obtain simultaneously upper bounds and lower bounds on the noise correlations. Combination of these bounds yields a lower bound on the probability of non-transitive outcome in terms of $D_{1}(F)$ or $D_{2}(F)$. 
3. To complete the proof, we use the techniques of Mossel to cover all the remaining cases (i.e., functions with $D_{1}(F)$ or $D_{2}(F)$ greater than a fixed constant, etc.)

We note that since in the case where $D_{1}(F)$ or $D_{2}(F)$ is greater than a fixed constant we use Mossel's result as a black box, the value of the constant we obtain in the dependence of $\delta(\epsilon)$ on $\epsilon$ is extremely low, and seems to be very far from optimality. Extension of our techniques to cover all the cases would make the proof free of non-linear invariance arguments, and lead to a decent value of the constant. This is one of the main open problems left in our paper.

This paper is organized as follows: In Section 2 we present the tools used in the later sections. In Section 3 we prove our main lemma. We deduce Theorems 1.3 and 1.5 from the main lemma in Section 4. In Section 5 we discuss the tightness of our results. We conclude the paper with questions for further research in Section 6.

\section{Preliminaries}

In this section we present the tools used in the next sections. First we describe the FourierWalsh expansion of functions on the discrete cube. We continue with the noise operator and the hypercontractive inequalities of Bonami-Beckner and of Borell. Finally, we cite the statements from Mossel's proof of the quantitative Arrow theorem [18] that are used as a black box in our proof.

\subsection{Fourier-Walsh expansion of functions on the discrete cube}

Throughout the paper we consider the discrete cube $\Omega=\{0,1\}^{n}$, endowed with the uniform measure $\mu$. Elements of $\Omega$ are represented either by binary vectors of length $n$, or by subsets of $\{1, \ldots, n\}$. Denote the set of all real-valued functions on $\Omega$ by $X$. The inner product of functions $f, g \in X$ is defined as usual by

$$
\langle f, g\rangle=\mathbb{E}_{\mu}[f g]=\frac{1}{2^{n}} \sum_{x \in\{0,1\}^{n}} f(x) g(x) .
$$

The Rademacher functions $\left\{r_{i}\right\}_{i=1}^{n}$, defined as $r_{i}\left(x_{1}, \ldots, x_{n}\right)=2 x_{i}-1$, constitute an orthonormal system in $X$. Moreover, this system can be completed to an orthonormal basis in $X$ by defining

$$
r_{S}=\prod_{i \in S} r_{i}
$$

for all $S \subset\{1, \ldots, n\}$. Every function $f \in X$ can be represented by its Fourier expansion with respect to the system $\left\{r_{S}\right\}_{S \subset\{1, \ldots, n\}}$ :

$$
f=\sum_{S \subset\{1, \ldots, n\}}\left\langle f, r_{S}\right\rangle r_{S} .
$$

This representation is called the Fourier-Walsh expansion of $f$. The coefficients in this expansion are denoted by

$$
\hat{f}(S)=\left\langle f, r_{S}\right\rangle
$$


The Fourier-Walsh expansion allows one to adapt tools from classical harmonic analysis to the discrete setting, and to use them in the study of Boolean functions. Since the introduction of such analytic methods in the landmark 1988 paper of Kahn, Kalai, and Linial [13], they have been intensively studied, and led to applications in numerous fields, including combinatorics, theoretical computer science, social choice theory, mathematical physics, etc. (see, e.g., the survey [16]).

The most basic analytic tool we use is the Parseval identity, asserting that for all $f, g \in X$,

$$
\langle f, g\rangle=\sum_{S \subset\{1, \ldots, n\}} \hat{f}(S) \hat{g}(S)
$$

and in particular, $\sum_{S \subset\{1, \ldots, n\}} \hat{f}(S)^{2}=\|f\|_{2}^{2}$ for any $f \in X$.

The next simple tool we use is the close relation between the Fourier-Walsh expansions of a function and of its dual function.

Definition 2.1. Let $f:\{0,1\}^{n} \rightarrow\{0,1\}$. The dual function of $f$, which we denote by $\bar{f}:\{0,1\}^{n} \rightarrow\{0,1\}$, is defined by

$$
\bar{f}\left(x_{1}, \ldots, x_{n}\right)=1-f\left(1-x_{1}, \ldots, 1-x_{n}\right) .
$$

Claim 2.2. Consider the Fourier-Walsh expansions of a Boolean function $f$ and of its dual function $\bar{f}$. For any $S \subset\{1, \ldots, n\}$ with $|S| \geq 1$,

$$
\widehat{\bar{f}}(S)=(-1)^{|S|-1} \hat{f}(S) .
$$

The simple proof of the claim is omitted. We also use a variant of the dual function: $f^{\prime}(x)=1-\bar{f}(x)$, defined as

$$
f^{\prime}\left(x_{1}, \ldots, x_{n}\right)=f\left(1-x_{1}, \ldots, 1-x_{n}\right) .
$$

Similarly to Claim 2.2, it is easy to see that for any $|S| \geq 1$,

$$
\widehat{f^{\prime}}(S)=(-1)^{|S|} \hat{f}(S) \text {. }
$$

In Kalai's proof of the quantitative Arrow theorem for neutral GSWFs [14], only the most basic analytic tools (like the Parseval identity) were used. Following the proof of Mossel [18], we use also more advanced analytic tools, related to the noise operator presented below.

\subsection{The noise operator and hypercontractive inequalities}

The noise operator, defined in [3, 4], is a convolution operator that represents the application of the function on a slightly perturbed input.

Definition 2.3. For $x \in\{0,1\}^{n}$, the $\epsilon$-noise perturbation of $x$, denoted by $N_{\epsilon}(x)$, is a distribution obtained from $x$ by independently keeping each coordinate of $x$ unchanged with probability $1-\epsilon$, and replacing it by a random value with probability $\epsilon$. 
Definition 2.4. Let $f:\{0,1\}^{n} \rightarrow\{0,1\}$. For $0 \leq \epsilon \leq 1$, the noise operator $T_{\epsilon}$ applied to $f$ is defined by

$$
T_{\epsilon} f(x)=\mathbb{E}_{y \sim N_{1-\epsilon}(x)}[f(y)] .
$$

It is easy to see that the noise operator has a convenient representation in terms of the Fourier-Walsh expansion:

Claim 2.5. Consider a function $f$ on the discrete cube with a Fourier-Walsh expansion $f=\sum_{S} \hat{f}(S) r_{S}$. The Fourier-Walsh expansion of $T_{\epsilon} f$ is given by

$$
T_{\epsilon} f=\sum_{S} \epsilon^{|S|} \hat{f}(S) r_{S}
$$

Since $T_{\epsilon} f$ represents the application of $f$ on a noisy variant of the input, it makes sense to define the $\epsilon$-noise correlation of two functions $f$ and $g$ as $\left\langle T_{\epsilon} f, g\right\rangle$. Using the Parseval identity, we get an equivalent definition in terms of the Fourier-Walsh expansion (note that the definition is symmetric between $f$ and $g$ ):

Definition 2.6. Given two functions $f, g:\{0,1\}^{n} \rightarrow\{0,1\}$, the $\epsilon$-noise correlation of $f, g$ is

$$
\left\langle T_{\epsilon} f, g\right\rangle=\sum_{S \subset\{1, \ldots, n\}} \epsilon^{|S|} \hat{f}(S) \hat{g}(S) .
$$

In the proof of Lemma 3.2 below, we express the probability of non-transitive outcome in a GSWF $F$ on three alternatives in terms of the noise correlations between the Boolean choice functions $F_{12}, F_{23}, F_{13}$. Then we obtain upper and lower bounds on the noise correlations using the hypercontractive inequalities presented below.

The first hypercontractive inequality we use is the Bonami-Beckner inequality, discovered independently by Bonami [4] in 1970 and by Beckner [3] in 1975.

Theorem 2.7 (Bonami, Beckner). Let $f:\{0,1\}^{n} \rightarrow \mathbb{R}$, and let $q_{1} \geq q_{2} \geq 1$. Then

$$
\left\|T_{\epsilon} f\right\|_{q_{1}} \leq\|f\|_{q_{2}} \quad \text { for all } 0 \leq \epsilon \leq\left(\frac{q_{2}-1}{q_{1}-1}\right)^{1 / 2} .
$$

In particular,

$$
\left\|T_{\epsilon} f\right\|_{2} \leq\|f\|_{1+\epsilon^{2}} \quad \text { for all } 0 \leq \epsilon \leq 1 .
$$

This inequality was first applied in a combinatorial context in [13], and since then it has been used in numerous papers in the field. We combine the Bonami-Beckner inequality with the Cauchy-Schwarz inequality to obtain an upper bound on the $\epsilon$-noise correlation of Boolean functions. The upper bound is presented here for $\epsilon=1 / 3$ since this is the case we use in the proof of Lemma 3.2, but it can be immediately generalized to any $0 \leq \epsilon \leq 1$.

Proposition 2.8. Let $f, g:\{0,1\}^{n} \rightarrow\{0,1\}$, and denote $\mathbb{E}[f]=p_{1}$ and $\mathbb{E}[g]=p_{2}$. Then

$$
\sum_{S}\left(\frac{1}{3}\right)^{|S|} \hat{f}(S) \hat{g}(S) \leq \min \left(p_{1}^{0.9} p_{2}^{0.5}, p_{1}^{0.75} p_{2}^{0.75}\right) .
$$


Proof. By Claim 2.5, the Parseval identity, the Cauchy-Schwarz inequality and the Bonami-Beckner hypercontractive inequality, we get

$$
\sum_{S}\left(\frac{1}{3}\right)^{|S|} \hat{f}(S) \hat{g}(S)=\left\langle T_{1 / 3} f, g\right\rangle \leq\left\|T_{1 / 3} f\right\|_{2}\|g\|_{2} \leq\|f\|_{1+1 / 9}\|g\|_{2}=p_{1}^{0.9} p_{2}^{0.5} .
$$

Similarly,

$$
\begin{aligned}
\sum_{S}\left(\frac{1}{3}\right)^{|S|} \hat{f}(S) \hat{g}(S) & =\left\langle T_{(1 / \sqrt{3})} f, T_{(1 / \sqrt{3})} g\right\rangle \leq\left\|T_{(1 / \sqrt{3})} f\right\|_{2}\left\|T_{(1 / \sqrt{3})} g\right\|_{2} \\
& \leq\|f\|_{1+1 / 3}\|g\|_{1+1 / 3}=p_{1}^{0.75} p_{2}^{0.75} .
\end{aligned}
$$

The second hypercontractive inequality we use is a reverse hypercontractive inequality, due to Borell [5]. This inequality asserts that under some conditions, a variant of the Bonami-Beckner inequality holds in the inverse direction.

Theorem 2.9 (Borell). Let $f:\{0,1\}^{n} \rightarrow \mathbb{R}_{+}$, and let $q_{1} \leq q_{2} \leq 1$. Then

$$
\left\|T_{\epsilon} f\right\|_{q_{1}} \geq\|f\|_{q_{2}} \quad \text { for all } 0 \leq \epsilon \leq\left(\frac{q_{2}-1}{q_{1}-1}\right)^{1 / 2} .
$$

Although Borell's result dates back to 1982, it was not used in the research of Boolean functions until recent years. In the last few years, Borell's inequality was used in several papers $[7,19,18]$; it seems to be a useful tool that has yet to be fully developed.

We use Borell's inequality to obtain a lower bound on the $\epsilon$-noise correlation of Boolean functions through the following corollary, presented in [19, Corollary 3.5]:

Theorem 2.10. Let $f, g:\{0,1\}^{n} \rightarrow\{0,1\}$. If $\mathbb{E}[f]=p_{1}$, and $\mathbb{E}[g]=p_{2}=p_{1}^{\alpha}$ for some $\alpha \geq 0$, then for all $0<\epsilon<1$,

$$
\sum_{S} \epsilon^{|S|} \hat{f}(S) \hat{g}(S) \geq p_{1} \cdot p_{1}^{(\sqrt{\alpha}+\epsilon)^{2} /\left(1-\epsilon^{2}\right)}=p_{1} \cdot p_{2}^{\frac{(\sqrt{\alpha}+\epsilon)^{2}}{\left(1-\epsilon^{2}\right) \alpha}} .
$$

In the proof of Lemma 3.2, we apply Theorem 2.10 with $\alpha \geq 1$, and write the lower bound in the form $p_{1} \cdot p_{2}^{\beta}$. We use a simple observation regarding properties of $\beta=$ $(\sqrt{\alpha}+\epsilon)^{2} /\left(\left(1-\epsilon^{2}\right) \alpha\right)$ :

Observation 2.11. - As a function of $\alpha=\log _{p_{1}}\left(p_{2}\right), \beta$ is decreasing.

- For all $\alpha \geq 1$, we have $\beta \leq \frac{1+\epsilon}{1-\epsilon}$. When $\alpha \rightarrow \infty$, we have $\beta \rightarrow \frac{1}{1-\epsilon^{2}}$.

Finally, we use the following notation:

Notation 2.12. We denote by $\operatorname{RHC}\left(p_{1}, p_{2}\right)$ the lower bound obtained in Theorem 2.10 for $\mathbb{E}[f]=p_{1}, \mathbb{E}[g]=p_{2}$, and $\epsilon=1 / 3$. In particular, $\operatorname{RHC}(p, p)=p^{3}$, and

$$
\operatorname{RHC}(1 / 2, p)=\frac{1}{2} \cdot p^{\frac{9(\sqrt{\alpha}+1 / 3)^{2}}{8 \alpha}},
$$

where $\alpha=\log _{2}(1 / p)$. For a small value of $p$, the exponent tends to $9 / 8$. 


\subsection{Mossel's quantitative Arrow theorem}

In the proofs of Theorems 1.3 and 1.5 we use (as a "black box") three major components of Mossel's proof of his quantitative version of Arrow's theorem [18]. The first is a quantitative Arrow theorem for GSWFs on three alternatives:

Theorem 2.13 ([18, Theorem 8.1]). There exists an absolute constant $C$ such that for any GSWF F on three alternatives that satisfies the IIA condition, if the probability of non-transitive outcome in $F$ is at most

$$
\delta(\epsilon)=\exp \left(-C / \epsilon^{21}\right)
$$

then $D_{1}(F) \leq \epsilon$.

We use this theorem only in the case where $\epsilon$ is greater than some fixed constant. Thus, the "bad" dependence of $\delta$ on $\epsilon$ affects our final result only by a constant factor.

The second component is a generic reduction lemma that allows extending results from GSWFs on three alternatives to GSWFs on $k$ alternatives, for all $k \geq 3$. The reduction can be formulated as follows:

Theorem 2.14 ([18, Theorem 9.1 and Remark 9.2]). Suppose that there exists $\delta_{0}(\epsilon)$ such that for any GSWF F on three alternatives that satisfies the IIA condition, if the probability of non-transitive outcome in $F$ is at most $\delta_{0}(\epsilon)$, then $D_{1}(F) \leq \epsilon$. Then we have the following quantitative Arrow theorem for GSWFs on $k$ alternatives: For any GSWF $F$ on $k$ alternatives that satisfies the IIA condition, if the probability of non-transitive outcome in $F$ is at most

$$
\delta(\epsilon)=\delta_{0}\left(\epsilon / k^{2}\right)
$$

then $D_{1}(F) \leq \epsilon$.

The third component is a complete characterization of the set $\mathcal{F}_{k}(n)$ of GSWFs on $k$ alternatives that satisfy the IIA condition and whose output is always transitive. Though we use in our proof only the characterization of $\mathcal{F}_{3}(n)$, the result is presented here for a general $k$ for the sake of completeness.

Theorem 2.15 ([18, Theorem 1.2]). The class $\mathcal{F}_{k}(n)$ consists exactly of all GSWFs $F$ satisfying the following: There exists a partition of the set of alternatives into disjoint sets $A_{1}, \ldots, A_{r}$ such that:

- For any profile, $F$ ranks all the alternatives in $A_{i}$ above all the alternatives in $A_{j}$, for all $i<j$.

- For all $s$ such that $\left|A_{s}\right| \geq 3$, the restriction of $F$ to the alternatives in $A_{s}$ is a dictatorship (i.e., is either the preference order of some voter $j$, or the reverse of such order).

- For all s such that $\left|A_{s}\right|=2$, the restriction of $F$ to the alternatives in $A_{s}$ is an arbitrary non-constant function of the individual preferences between the two alternatives in $A_{s}$. 


\section{Proof of the main lemma}

In this section we prove our main lemma, asserting that if $F$ is a GSWF on three alternatives that satisfies the IIA condition, and at least one of the Boolean choice functions $F_{12}, F_{23}, F_{31}$ is close enough to a constant function, then the probability of non-transitive outcome can be bounded from below in terms of $D_{1}(F)$ and $D_{2}(F)$. Throughout this section we use the following notation:

Notation 3.1. The Boolean choice functions $F_{12}, F_{23}, F_{31}$ are denoted by $f, g, h$, respectively. This means that the preferences of the voters between alternatives 1 and 2 are denoted by a vector $\left(x_{1}, \ldots, x_{n}\right) \in\{0,1\}^{n}$, where $x_{k}=1$ if the $k$ th voter prefers alternative 1 over alternative 2 , and $x_{k}=0$ otherwise. Then $f\left(x_{1}, \ldots, x_{n}\right)=1$ if in the output of $F$, alternative 1 is preferred over alternative 2 , and $f\left(x_{1}, \ldots, x_{n}\right)=0$ otherwise. The functions $g$ and $h$ are defined similarly with respect to the pairs of alternatives $(2,3)$ and $(3,1)$. The expectations of the choice functions are denoted by

$$
\mathbb{E}[f]=p_{1}, \quad \mathbb{E}[g]=p_{2}, \quad \mathbb{E}[h]=p_{3} .
$$

For each $i$, we denote $\bar{p}_{i}=\min \left(p_{i}, 1-p_{i}\right)$, and let

$$
D_{2}^{\prime}(F)=\min _{1 \leq i \leq 3} \bar{p}_{i}
$$

Note that $D_{2}^{\prime}(F)$ measures the distance of the Boolean choice functions of $F$ from the family of constant functions. Finally, the probability of non-transitive outcome is denoted by $P(F)$.

Now we can formulate our main lemma:

Lemma 3.2. Let $F$ be a GSWF on three alternatives satisfying the IIA condition. If $D_{2}^{\prime}(F) \leq 2^{-500000}$, then

$$
P(F) \geq \frac{1}{10} \cdot \max \left(\operatorname{RHC}\left(D_{1}(F) / 2, D_{1}(F) / 2\right), \operatorname{RHC}\left(D_{2}^{\prime}(F), 1 / 2\right)\right),
$$

where $D_{1}(F)$ is as defined in the introduction and $\mathrm{RHC}(\cdot, \cdot)$ is as in Notation 2.12.

Proof. Our starting point is Kalai's formula [14] for the probability of non-transitive outcome in a GSWF on three alternatives satisfying the IIA condition:

$$
\begin{aligned}
P(F)= & p_{1} p_{2} p_{3}+\left(1-p_{1}\right)\left(1-p_{2}\right)\left(1-p_{3}\right)+\sum_{S \neq \emptyset}\left(-\frac{1}{3}\right)^{|S|} \hat{f}(S) \hat{g}(S) \\
& +\sum_{S \neq \emptyset}\left(-\frac{1}{3}\right)^{|S|} \hat{g}(S) \hat{h}(S)+\sum_{S \neq \emptyset}\left(-\frac{1}{3}\right)^{|S|} \hat{h}(S) \hat{f}(S) .
\end{aligned}
$$

The proof is divided into several cases, and in each case we use a different modification of formula (9). In the following, we assume without loss of generality that $\bar{p}_{1} \leq \bar{p}_{2} \leq \bar{p}_{3}$. Moreover, we assume that $p_{1} \leq 1 / 2$, since otherwise we can replace $f, g, h$ by the dual functions without changing the value of the right hand side of (9). 


\subsection{Case 1: $p_{1}, p_{2} \leq 1 / 2$}

First, we note that if $p_{3} \leq 1 / 2$, then the assertion follows easily from Kalai's formula (9). Indeed, since by assumption $p_{1}<2^{-500000}$, it follows that $\left(1-p_{1}\right)\left(1-p_{2}\right)\left(1-p_{3}\right) \geq$ $\frac{1}{4}\left(1-2^{-500000}\right)$. On the other hand, by the Parseval identity and the Cauchy-Schwarz inequality, we have

$$
\left|\sum_{S \neq \emptyset}\left(-\frac{1}{3}\right)^{|S|} \hat{f}(S) \hat{g}(S)\right| \leq \frac{1}{3}\left(\langle f, g\rangle-p_{1} p_{2}\right) \leq \frac{1}{3} \cdot \frac{1}{2} \cdot 2^{-250000},
$$

and similarly,

$$
\left|\sum_{S \neq \emptyset}\left(-\frac{1}{3}\right)^{|S|} \hat{g}(S) \hat{h}(S)\right| \leq \frac{1}{3} \cdot \frac{1}{2} \cdot \frac{1}{2} \quad \text { and }\left|\sum_{S \neq \emptyset}\left(-\frac{1}{3}\right)^{|S|} \hat{h}(S) \hat{f}(S)\right| \leq \frac{1}{3} \cdot \frac{1}{2} \cdot 2^{-250000} \text {. }
$$

Thus, by (9),

$$
P(F) \geq \frac{1}{4}\left(1-2^{-500000}\right)-\frac{1}{12}-2 \cdot \frac{1}{3} \cdot \frac{1}{2} \cdot 2^{-250000}>1 / 10 .
$$

Therefore, we can assume that $p_{3} \geq 1 / 2$. Note that in this case, we have

$$
D_{1}(F) \leq 2\left(1-p_{3}\right) \text {. }
$$

Indeed, define a GSWF $G$ on three alternatives by the choice functions

$$
f^{\prime}=G_{12}=0 \text { (constant), } \quad g^{\prime}=G_{23}=g, \quad h^{\prime}=G_{31}=1 \text { (constant). }
$$

It is clear that $G \in \mathcal{F}_{3}(n)$, since $G$ always ranks alternative 1 at the bottom and thus its output is always transitive, and

$$
\operatorname{Pr}[F \neq G] \leq \operatorname{Pr}\left[f \neq f^{\prime}\right]+\operatorname{Pr}\left[g \neq g^{\prime}\right]+\operatorname{Pr}\left[h \neq h^{\prime}\right] \leq p_{1}+\left(1-p_{3}\right) \leq 2\left(1-p_{3}\right) .
$$

Therefore, $D_{1}(F) \leq D(F, G) \leq 2\left(1-p_{3}\right)$. Also, by the definition,

$$
D_{2}^{\prime}(F)=p_{1} \text {. }
$$

We modify formula (9) using the following identities:

$$
\begin{aligned}
\sum_{S \neq \emptyset}\left(-\frac{1}{3}\right)^{|S|} \hat{f}(S) \hat{g}(S) & =\sum_{S \neq \emptyset}\left(\frac{1}{3}\right)^{|S|} \hat{f^{\prime}}(S) \hat{g}(S)=\left\langle T_{1 / 3} f^{\prime}, g\right\rangle-p_{1} p_{2} \\
\sum_{S \neq \emptyset}\left(-\frac{1}{3}\right)^{|S|} \hat{g}(S) \hat{h}(S) & =\sum_{S \neq \emptyset}\left(-\frac{1}{3}\right)^{|S|} \widehat{\bar{g}}(S) \widehat{\bar{h}}(S)=\sum_{S \neq \emptyset}\left(\frac{1}{3}\right)^{|S|} \widehat{\bar{g}}(S) \widehat{1-h}(S) \\
& =\left\langle T_{1 / 3} \bar{g}, 1-h\right\rangle-\left(1-p_{2}\right)\left(1-p_{3}\right) . \\
\sum_{S \neq \emptyset}\left(-\frac{1}{3}\right)^{|S|} \hat{h}(S) \hat{f}(S) & =\left\langle T_{1 / 3} f^{\prime}, h\right\rangle-p_{1} p_{3}=\left\langle T_{1 / 3} f^{\prime}, 1\right\rangle-\left\langle T_{1 / 3} f^{\prime}, 1-h\right\rangle-p_{1} p_{3} \\
& =p_{1}-p_{1} p_{3}-\left\langle T_{1 / 3} f^{\prime}, 1-h\right\rangle .
\end{aligned}
$$


All three identities follow immediately from basic properties of the dual function (e.g., (2) and (3)) and the Parseval identity. Substituting (12)-(14) into (9), we get

$$
\begin{aligned}
P(F)= & p_{1} p_{2} p_{3}+\left(1-p_{1}\right)\left(1-p_{2}\right)\left(1-p_{3}\right)+\left\langle T_{1 / 3} f^{\prime}, g\right\rangle-p_{1} p_{2} \\
& +\left\langle T_{1 / 3} \bar{g}, 1-h\right\rangle-\left(1-p_{2}\right)\left(1-p_{3}\right)+p_{1}-p_{1} p_{3}-\left\langle T_{1 / 3} f^{\prime}, 1-h\right\rangle \\
= & \left\langle T_{1 / 3} f^{\prime}, g\right\rangle+\left\langle T_{1 / 3} \bar{g}, 1-h\right\rangle-\left\langle T_{1 / 3} f^{\prime}, 1-h\right\rangle .
\end{aligned}
$$

Equation (15) expresses $P(F)$ as a linear combination of noise correlations between the functions $f, g, h$, which are obviously non-negative. Thus, if we obtain a lower bound on the noise correlations that appear in (15) with a ' + ' sign, and an upper bound on the correlation that appears with a '-' sign, we will get a lower bound on $P(f)$. We shall obtain these bounds using the Bonami-Beckner hypercontractive inequality and Borell's reverse hypercontractive inequality. We subdivide our proof into two subcases.

3.1.1. Case $1 a: 1-p_{3}<1 / 32$. We bound $\left\langle T_{1 / 3} f^{\prime}, 1-h\right\rangle$ from above using the BonamiBeckner hypercontractive inequality. By Proposition 2.8, we get

$$
\left\langle T_{1 / 3} f^{\prime}, 1-h\right\rangle \leq p_{1}^{0.75}\left(1-p_{3}\right)^{0.75} \leq\left(1-p_{3}\right)^{1.5} \text {. }
$$

We bound $\left\langle T_{1 / 3} \bar{g}, 1-h\right\rangle$ from below using Borell's reverse hypercontractive inequality. By Theorem 2.10, we have

$$
\left\langle T_{1 / 3} \bar{g}, 1-h\right\rangle \geq \operatorname{RHC}\left(1-p_{2}, 1-p_{3}\right) .
$$

In order to estimate $\operatorname{RHC}\left(1-p_{2}, 1-p_{3}\right)$, we write it in the form $\left(1-p_{2}\right) \cdot\left(1-p_{3}\right)^{\beta(\alpha)}$, where $\alpha=\log _{1-p_{2}}\left(1-p_{3}\right)$. By Observation 2.11, $\beta(\alpha)$ is a decreasing function of $\alpha$. Since by assumption, $1-p_{2}>31 / 32$ and $1-p_{3}<1 / 32$, we have $\alpha \geq \log _{31 / 32}(1 / 32)=$ 109.16. Substituting the value $\alpha=109.16$ into the definition of $\beta(\alpha)$ and using the monotonicity of $\beta(\alpha)$, we get $\beta \leq 1.198$, and thus

$$
\left\langle T_{1 / 3} \bar{g}, 1-h\right\rangle \geq\left(1-p_{2}\right)\left(1-p_{3}\right)^{1.198} \geq \frac{31}{32}\left(1-p_{3}\right)^{1.198} .
$$

Combining inequalities (16) and (17), we get

$$
\begin{aligned}
\left\langle T_{1 / 3} f^{\prime}, 1-h\right\rangle \leq\left(1-p_{3}\right)^{1.5} & =\left(\frac{32}{31}\left(1-p_{3}\right)^{0.302}\right)\left(\frac{31}{32}\left(1-p_{3}\right)^{1.198}\right) \\
& \leq\left(\frac{32}{31}\left(\frac{1}{32}\right)^{0.302}\right)\left\langle T_{1 / 3} \bar{g}, 1-h\right\rangle \leq 0.37\left\langle T_{1 / 3} \bar{g}, 1-h\right\rangle .
\end{aligned}
$$

Finally, substituting into (15) we get

$$
\begin{aligned}
P(F) & =\left\langle T_{1 / 3} f^{\prime}, g\right\rangle+\left\langle T_{1 / 3} \bar{g}, 1-h\right\rangle-\left\langle T_{1 / 3} f^{\prime}, 1-h\right\rangle \\
& \geq\left\langle T_{1 / 3} \bar{g}, 1-h\right\rangle-\left\langle T_{1 / 3} f^{\prime}, 1-h\right\rangle \geq 0.63\left\langle T_{1 / 3} \bar{g}, 1-h\right\rangle \\
& \geq 0.63 \operatorname{RHC}\left(1-p_{2}, 1-p_{3}\right) \geq 0.63 \max \left(\operatorname{RHC}\left(1-p_{3}, 1-p_{3}\right), \operatorname{RHC}\left(1 / 2, p_{1}\right)\right),
\end{aligned}
$$

where the last inequality holds since $1-p_{2} \geq 1 / 2 \geq 1-p_{3} \geq p_{1}$ and since $\operatorname{RHC}(\cdot, \cdot)$ is clearly non-decreasing in its arguments. The assertion of the lemma now follows from (10) and (11). 
3.1.2. Case $1 b: 1-p_{3} \geq 1 / 32$. As in the previous case, we bound $\left\langle T_{1 / 3} f^{\prime}, 1-h\right\rangle$ from above using the Bonami-Beckner hypercontractive inequality. By Proposition 2.8, we get

$$
\left\langle T_{1 / 3} f^{\prime}, 1-h\right\rangle \leq p_{1}^{0.9}\left(1-p_{3}\right)^{0.5} \leq\left(1-p_{3}\right)^{4.1},
$$

where the last inequality follows since by assumption $p_{1} \leq 2^{-500000}$, and in particular $p_{1} \leq\left(1-p_{3}\right)^{4}$. In order to bound $\left\langle T_{1 / 3} \bar{g}, 1-h\right\rangle$ from below we use the reverse hypercontractive inequality. By Theorem 2.10, we have

$$
\left\langle T_{1 / 3} \bar{g}, 1-h\right\rangle \geq \operatorname{RHC}\left(1-p_{2}, 1-p_{3}\right) .
$$

As in the previous case, we write RHC $\left(1-p_{2}, 1-p_{3}\right)$ in the form $\left(1-p_{2}\right) \cdot\left(1-p_{3}\right)^{\beta(\alpha)}$. Since by Observation 2.11, for any $\alpha \geq 1$, we have $\beta(\alpha) \leq \frac{1+\epsilon}{1-\epsilon}=2$, we get

$$
\left\langle T_{1 / 3} \bar{g}, 1-h\right\rangle \geq\left(1-p_{2}\right)\left(1-p_{3}\right)^{2} \geq 0.5\left(1-p_{3}\right)^{2} .
$$

Combination of (18) and (19) yields

$$
\begin{aligned}
\left\langle T_{1 / 3} f^{\prime}, 1-h\right\rangle \leq\left(1-p_{3}\right)^{4.1} & =\left(2\left(1-p_{3}\right)^{2.1}\right)\left(0.5\left(1-p_{3}\right)^{2}\right) \\
& \leq 2\left(1-p_{3}\right)^{2.1}\left\langle T_{1 / 3} \bar{g}, 1-h\right\rangle \leq 0.5\left\langle T_{1 / 3} \bar{g}, 1-h\right\rangle,
\end{aligned}
$$

where the last inequality follows since $1-p_{3} \leq 1 / 2$. Finally,

$$
\begin{aligned}
P(F) & =\left\langle T_{1 / 3} f^{\prime}, g\right\rangle+\left\langle T_{1 / 3} \bar{g}, 1-h\right\rangle-\left\langle T_{1 / 3} f^{\prime}, 1-h\right\rangle \\
& \geq\left\langle T_{1 / 3} \bar{g}, 1-h\right\rangle-\left\langle T_{1 / 3} f^{\prime}, 1-h\right\rangle \geq 0.5\left\langle T_{1 / 3} \bar{g}, 1-h\right\rangle \\
& \geq 0.5 \operatorname{RHC}\left(1-p_{2}, 1-p_{3}\right) \\
& \geq 0.5 \max \left(\operatorname{RHC}\left(1-p_{3}, 1-p_{3}\right), \operatorname{RHC}\left(1 / 2, p_{1}\right)\right),
\end{aligned}
$$

as asserted. This completes the proof of Case 1.

3.2. Case 2: $p_{1} \leq 1 / 2$ and $p_{2} \geq 1 / 2$

In this case, we have

$$
D_{1}(F) \leq 2\left(1-p_{2}\right),
$$

since defining a GSWF $G^{\prime}$ on three alternatives by the choice functions

$$
f^{\prime \prime}=G_{12}^{\prime}=0 \text { (constant), } \quad g^{\prime \prime}=G_{23}^{\prime}=1 \text { (constant) }, \quad h^{\prime \prime}=G_{31}^{\prime}=h,
$$

we get $G^{\prime} \in \mathcal{F}_{3}(n)$, and $D\left(F, G^{\prime}\right) \leq 2\left(1-p_{2}\right)$. Also, it is clear that as in Case 1,

$$
D_{2}(F) \leq D(f, \text { const })=p_{1} .
$$

This time we use a slightly different modification of Kalai's formula. Specifically, we interchange the roles of $g$ and $h$ in (12)-(14) to get the following modification of (15):

$$
P(F)=\left\langle T_{1 / 3} f^{\prime}, h\right\rangle+\left\langle T_{1 / 3}(1-g), \bar{h}\right\rangle-\left\langle T_{1 / 3} f^{\prime}, 1-g\right\rangle .
$$

We subdivide the argument into several subcases. 
3.2.1. Case $2 a: 1-p_{2} \leq p_{1}^{0.45412}$. By Proposition 2.8, we get

$$
\left\langle T_{1 / 3} f^{\prime}, 1-g\right\rangle \leq p_{1}^{0.9}\left(1-p_{2}\right)^{0.5} \leq p_{1}^{0.9} p_{1}^{0.22706}=p_{1}^{1.12706} .
$$

On the other hand, by Theorem 2.10 , we have

$$
\left\langle T_{1 / 3} f^{\prime}, h\right\rangle+\left\langle T_{1 / 3}(1-g), \bar{h}\right\rangle \geq \operatorname{RHC}\left(p_{3}, p_{1}\right)+\operatorname{RHC}\left(1-p_{3}, 1-p_{2}\right) .
$$

Since $p_{1} \leq 1-p_{2}$ and either $p_{3}$ or $1-p_{3}$ is not less than $1 / 2$, we get

$$
\left\langle T_{1 / 3} f^{\prime}, h\right\rangle+\left\langle T_{1 / 3}(1-g), \bar{h}\right\rangle \geq \operatorname{RHC}\left(1 / 2, p_{1}\right) \geq 0.5 p_{1}^{1.12606},
$$

where the last inequality follows from Observation 2.11 since $p_{1} \leq 2^{-500000}$. Combining (23) and (24) we get

$\left\langle T_{1 / 3} f^{\prime}, 1-g\right\rangle \leq p_{1}^{1.12706}=\left(2 p_{1}^{0.001}\right)\left(0.5 p_{1}^{1.12606}\right) \leq 0.5\left(\left\langle T_{1 / 3} f^{\prime}, h\right\rangle+\left\langle T_{1 / 3}(1-g), \bar{h}\right\rangle\right)$.

Finally,

$$
\begin{aligned}
P(F) & =\left\langle T_{1 / 3} f^{\prime}, h\right\rangle+\left\langle T_{1 / 3}(1-g), \bar{h}\right\rangle-\left\langle T_{1 / 3} f^{\prime}, 1-g\right\rangle \\
& \geq 0.5\left(\left\langle T_{1 / 3} f^{\prime}, h\right\rangle+\left\langle T_{1 / 3}(1-g), \bar{h}\right\rangle\right) \\
& \geq 0.5\left(\operatorname{RHC}\left(p_{3}, p_{1}\right)+\operatorname{RHC}\left(1-p_{3}, 1-p_{2}\right)\right) \\
& \geq 0.5 \max \left(\operatorname{RHC}\left(1-p_{2}, 1-p_{2}\right), \operatorname{RHC}\left(1 / 2, p_{1}\right)\right),
\end{aligned}
$$

where the last inequality holds since $1-p_{3} \geq 1-p_{2}$. The assertion of the lemma follows now from (20) and (21).

3.2.2. Case $2 b: 1-p_{2}>p_{1}^{0.45412}$ and $\bar{p}_{3} \geq p_{1}^{0.2002}$. The upper bound in this case is the same as in Case 2a:

$$
\left\langle T_{1 / 3} f^{\prime}, 1-g\right\rangle \leq p_{1}^{0.9}\left(1-p_{2}\right)^{0.5} .
$$

For the lower bound, we use the reverse hypercontractive inequality for the term $\left\langle T_{1 / 3}(1-g), \bar{h}\right\rangle$ to get

$$
\left\langle T_{1 / 3}(1-g), \bar{h}\right\rangle \geq \operatorname{RHC}\left(1-p_{3}, 1-p_{2}\right) \geq\left(1-p_{3}\right)\left(1-p_{2}\right)^{2} \geq p_{1}^{0.2002}\left(1-p_{2}\right)^{2},
$$

where the second inequality follows from Observation 2.11, and the third from the assumption $\bar{p}_{3} \geq p_{1}^{0.2002}$. Combination of (25) and (26) yields

$$
\begin{aligned}
\left\langle T_{1 / 3} f^{\prime}, 1-g\right\rangle & \leq p_{1}^{0.9} p_{2}^{0.5}=\left(p_{1}^{0.6998} p_{2}^{-1.5}\right)\left(p_{1}^{0.2002}\left(1-p_{2}\right)^{2}\right) \\
& \leq p_{1}^{0.01862}\left\langle T_{1 / 3}(1-g), \bar{h}\right\rangle \leq 0.5\left\langle T_{1 / 3}(1-g), \bar{h}\right\rangle,
\end{aligned}
$$

where the second last inequality follows from the assumption $1-p_{2} \geq p_{1}^{0.45412}$, and the last from $p_{1} \leq 2^{-500000}$. Finally, if $p_{3} \geq 1 / 2$ then $\left\langle T_{1 / 3} f^{\prime}, h\right\rangle \geq \operatorname{RHC}\left(1 / 2, p_{1}\right)$, and otherwise $\left\langle T_{1 / 3}(1-g), \bar{h}\right\rangle \geq \operatorname{RHC}\left(1 / 2, p_{1}\right)$. In both cases,

$$
\begin{aligned}
P(F) & =\left\langle T_{1 / 3} f^{\prime}, h\right\rangle+\left(\left\langle T_{1 / 3}(1-g), \bar{h}\right\rangle-\left\langle T_{1 / 3} f^{\prime}, 1-g\right\rangle\right) \\
& \geq\left\langle T_{1 / 3} f^{\prime}, h\right\rangle+0.5\left\langle T_{1 / 3}(1-g), \bar{h}\right\rangle \\
& \geq 0.5 \max \left(\operatorname{RHC}\left(1-p_{2}, 1-p_{2}\right), \operatorname{RHC}\left(1 / 2, p_{1}\right)\right),
\end{aligned}
$$

and the assertion follows. 
3.2.3. Case $2 c: p_{3} \leq p_{1}^{0.2002}$. In this case we use another modification of Kalai's formula (9), resulting from the following modification of equation (22):

$$
\begin{aligned}
P(F) & =\left\langle T_{1 / 3} f^{\prime}, h\right\rangle+\left\langle T_{1 / 3}(1-g), \bar{h}\right\rangle-\left\langle T_{1 / 3} f^{\prime}, 1-g\right\rangle \\
& =\left\langle T_{1 / 3} f^{\prime}, h\right\rangle+\left(\left\langle T_{1 / 3}(1-g), 1\right\rangle-\left\langle T_{1 / 3}(1-g), 1-\bar{h}\right\rangle\right)-\left\langle T_{1 / 3} f^{\prime}, 1-g\right\rangle \\
& =\left(1-p_{2}\right)+\left\langle T_{1 / 3} f^{\prime}, h\right\rangle-\left\langle T_{1 / 3}(1-g), 1-\bar{h}\right\rangle-\left\langle T_{1 / 3} f^{\prime}, 1-g\right\rangle .
\end{aligned}
$$

By Proposition 2.8,

$$
\left\langle T_{1 / 3}(1-g), 1-\bar{h}\right\rangle \leq\left(1-p_{2}\right)^{0.9} p_{3}^{0.5} \leq\left(1-p_{2}\right)^{0.9} p_{1}^{0.1001} \leq\left(1-p_{2}\right)^{1.0001} .
$$

Similarly,

$$
\left\langle T_{1 / 3} f^{\prime}, 1-g\right\rangle \leq p_{1}^{0.9}\left(1-p_{2}\right)^{0.5} \leq\left(1-p_{2}\right)^{1.4}
$$

Hence,

$$
\begin{aligned}
\left\langle T_{1 / 3}(1-g), 1-\bar{h}\right\rangle+\left\langle T_{1 / 3} f^{\prime}, 1-g\right\rangle & \leq\left(1-p_{2}\right)^{1.0001}+\left(1-p_{2}\right)^{1.4} \\
& \leq 2\left(1-p_{2}\right)^{1.0001} \leq 0.5\left(1-p_{2}\right),
\end{aligned}
$$

where the last inequality follows since

$$
\left(1-p_{2}\right)^{0.0001} \leq p_{3}^{0.0001} \leq p_{1}^{0.2002 \cdot 0.0001}<1 / 2
$$

Finally, by (27),

$$
\begin{aligned}
P(F) & \geq\left(1-p_{2}\right)-\left\langle T_{1 / 3}(1-g), 1-\bar{h}\right\rangle-\left\langle T_{1 / 3} f^{\prime}, 1-g\right\rangle \\
& \geq 0.5\left(1-p_{2}\right) \geq 0.5 \max \left(\operatorname{RHC}\left(1-p_{2}, 1-p_{2}\right), \operatorname{RHC}\left(1 / 2, p_{1}\right)\right),
\end{aligned}
$$

as asserted.

3.2.4. Case $2 d: 1-p_{3} \leq p_{1}^{0.2002}$. We use yet another modification of equation (22):

$$
\begin{aligned}
P(F) & =\left\langle T_{1 / 3} f^{\prime}, h\right\rangle+\left\langle T_{1 / 3}(1-g), \bar{h}\right\rangle-\left\langle T_{1 / 3} f^{\prime}, 1-g\right\rangle \\
& =\left(\left\langle T_{1 / 3} f^{\prime}, 1\right\rangle-\left\langle T_{1 / 3} f^{\prime}, 1-h\right\rangle\right)+\left\langle T_{1 / 3}(1-g), \bar{h}\right\rangle-\left\langle T_{1 / 3} f^{\prime}, 1-g\right\rangle \\
& =p_{1}-\left\langle T_{1 / 3} f^{\prime}, 1-h\right\rangle+\left\langle T_{1 / 3}(1-g), \bar{h}\right\rangle-\left\langle T_{1 / 3} f^{\prime}, 1-g\right\rangle .
\end{aligned}
$$

Similarly to Case $2 \mathrm{c}$, we have

$$
\left\langle T_{1 / 3} f^{\prime}, 1-h\right\rangle \leq p_{1}^{0.9}\left(1-p_{3}\right)^{0.5} \leq p_{1}^{1.0001},
$$

and $\left\langle T_{1 / 3} f^{\prime}, 1-g\right\rangle \leq p_{1}^{1.4}$, and thus

$$
\left\langle T_{1 / 3} f^{\prime}, 1-h\right\rangle+\left\langle T_{1 / 3} f^{\prime}, 1-g\right\rangle \leq p_{1}^{1.0001}+p_{1}^{1.4} \leq 0.5 p_{1} .
$$

Therefore,

$$
\begin{aligned}
P(F) & =p_{1}-\left\langle T_{1 / 3} f^{\prime}, 1-h\right\rangle+\left\langle T_{1 / 3}(1-g), \bar{h}\right\rangle-\left\langle T_{1 / 3} f^{\prime}, 1-g\right\rangle \\
& \geq 0.5 p_{1}+\left\langle T_{1 / 3}(1-g), \bar{h}\right\rangle \geq 0.5 \max \left(\operatorname{RHC}\left(1-p_{2}, 1-p_{2}\right), \operatorname{RHC}\left(1 / 2, p_{1}\right)\right) .
\end{aligned}
$$

This completes the proof of Lemma 3.2. 


\section{Proofs of Theorems 1.3 and 1.5}

In this section we present the proofs of Theorems 1.3 and 1.5. The proofs are based on Lemma 3.2, but also rely heavily on several components of Mossel's proof of his quantitative version of Arrow's theorem cited in Section 2.3. The general structure of both proofs is as follows:

1. We consider first GSWFs on three alternatives, and examine several cases:

(a) If $D_{1}(F)$ (resp., $D_{2}(F)$ ) is greater than a fixed constant, we deduce the assertion from Theorem 2.13.

(b) If $F$ is close to a GSWF that always ranks one of the candidates at the top/bottom (resp., if at least one of the Boolean choice functions of $F$ is close to a constant function), we deduce the assertion from Lemma 3.2.

(c) If $F$ (resp., one of the Boolean choice functions of $F$ ) is close to a dictatorship of the $i$ th voter, we split $F$ into six GSWFs $\left\{F^{\sigma}\right\}_{\sigma \in S_{3}}$ according to the preferences of the $i$ th voter. We further subdivide this case into two subcases:

- If for all $\sigma \in S_{3}, D_{1}\left(F^{\sigma}\right)$ (resp., $D_{2}^{\prime}\left(F^{\sigma}\right)$ ) is small, we get a contradiction (resp., show directly that $P(F)$ cannot be small).

- If there exists $\sigma_{0} \in S_{3}$ such that $D_{1}\left(F^{\sigma_{0}}\right)$ (resp., $D_{2}^{\prime}\left(F^{\sigma_{0}}\right)$ ) is not small, we deduce the assertion by applying Lemma 3.2 to the GSWF $F^{\sigma_{0}}$.

2. We extend the result to GSWFs on $k$ alternatives, for all $k \geq 3$. In the proof of Theorem 1.3 this requires the reduction technique of Theorem 2.14, and in the proof of Theorem 1.5, the generalization is immediate.

Since the proofs differ in many of the details, we present them separately. Throughout this section, we use the notation defined at the beginning of Section 3.

\subsection{Proof of Theorem 1.3}

Theorem 4.1. There exists an absolute constant $C$ such that for any GSWF F on three alternatives that satisfies the IIA condition, if the probability of non-transitive outcome in $F$ is at most

$$
\delta(\epsilon)=\min \left(C, \frac{1}{50000} \cdot \epsilon^{3}\right),
$$

then $D_{1}(F) \leq \epsilon$.

Proof. It is clearly sufficient to prove that for any $\epsilon>0$, if $D_{1}(F)=\epsilon$, then $P(F) \geq$ $\min \left(C, \frac{1}{50000} \cdot \epsilon^{3}\right)$, for a universal constant $C$. We shall prove this for

$$
C=\exp \left(-\frac{C^{\prime}}{\left(2^{-500003}\right)^{21}}\right),
$$

where $C^{\prime}$ is the constant in Mossel's Theorem 2.13.

Let $F$ be a GSWF on three alternatives satisfying the IIA condition, and denote the

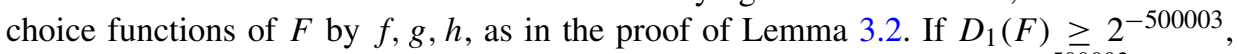
then by Theorem 2.13, $P(F) \geq C$. Thus, we may assume that $D_{1}(F)<2^{-500003}$. 
Let $G \in \mathcal{F}_{3}(n)$ satisfy $\operatorname{Pr}[F \neq G]=D_{1}(F)$ (such an element exists by the definition of the distance $D_{1}(F)$ ). Denote the Boolean choice functions of $G$ by $f^{\prime}, g^{\prime}, h^{\prime}$. By Theorem 2.15, $G$ either always ranks one alternative at the top/bottom or is a dictatorship. If $G$ always ranks one alternative at the top/bottom, then at least two of the functions $f^{\prime}, g^{\prime}, h^{\prime}$ are constant. Assume that $f^{\prime}$ and $g^{\prime}$ are constant. Since

$$
D_{1}(F)=\operatorname{Pr}[F \neq G] \geq \max \left(\operatorname{Pr}\left[f \neq f^{\prime}\right], \operatorname{Pr}\left[g \neq g^{\prime}\right], \operatorname{Pr}\left[h \neq h^{\prime}\right]\right),
$$

it follows that either $\mathbb{E}[f] \leq D_{1}(F)$ or $\mathbb{E}[f] \geq 1-D_{1}(F)$, and similarly for $g$. This implies that $D_{2}^{\prime}(F) \leq D_{1}(F)<2^{-500003}$, and thus we can apply Lemma 3.2 to $F$ and get

$$
P(F) \geq \frac{1}{10} \cdot \operatorname{RHC}\left(D_{1}(F) / 2, D_{1}(F) / 2\right) \geq \frac{1}{10} \cdot\left(D_{1}(F) / 2\right)^{3}>\frac{1}{50000} \cdot D_{1}(F)^{3},
$$

as asserted. Thus, we may assume that $G$ is a dictatorship.

The following part of the proof is similar to the proof of Theorem 7.1 in [18]. We can assume that the output of $G$ is determined by the first voter. We split the choice functions according to the first voter. Let

$$
f^{0}\left(x_{2}, \ldots, x_{n}\right)=f\left(0, x_{2}, \ldots, x_{n}\right), \quad f^{1}\left(x_{2}, \ldots, x_{n}\right)=f\left(1, x_{2}, \ldots, x_{n}\right),
$$

and similarly for $g$ and $h$. Furthermore, for any profile $\left(\sigma_{1}, \ldots, \sigma_{n}\right) \in S_{3}^{n}$, denote

$$
F^{\sigma_{1}}\left(\sigma_{2}, \ldots, \sigma_{n}\right)=F\left(\sigma_{1}, \sigma_{2}, \ldots, \sigma_{n}\right),
$$

and similarly for $G$. The Boolean choice functions of $F^{\sigma}$ are $f^{a_{1}}, g^{a_{2}}, h^{a_{3}}$, where $\left(a_{1}, a_{2}, a_{3}\right) \in\{0,1\}^{3}$ represents the preference $\sigma$ of the first voter (note that only six of the eight possible combinations of $\left(a_{1}, a_{2}, a_{3}\right)$ represent elements of $\left.S_{3}\right)$. Denote by $\bar{f}^{a_{1}}, \bar{g}^{a_{2}}, \bar{h}^{a_{3}}$ the choice functions of $G^{\sigma}$. Since $G$ is a dictatorship of the first voter, the functions $\bar{f}^{a_{1}}, \bar{g}^{a_{2}}, \bar{h}^{a_{3}}$ are constant. Clearly, we have

$$
D_{1}(F)=\operatorname{Pr}[F \neq G]=\frac{1}{6} \sum_{\sigma \in S_{3}} \operatorname{Pr}\left[F^{\sigma} \neq G^{\sigma}\right],
$$

and thus, for all $\sigma \in S_{3}$,

$$
\operatorname{Pr}\left[F^{\sigma} \neq G^{\sigma}\right] \leq 6 D_{1}(F)
$$

Since

$$
\operatorname{Pr}\left[F^{\sigma} \neq G^{\sigma}\right] \geq \max \left(\operatorname{Pr}\left[f^{a_{1}} \neq \bar{f}^{a_{1}}\right], \operatorname{Pr}\left[g^{a_{2}} \neq \bar{g}^{a_{2}}\right], \operatorname{Pr}\left[h^{a_{3}} \neq \bar{h}^{a_{3}}\right]\right),
$$

and since $G^{\sigma}$ is constant, this implies that

$$
\mathbb{E}\left[f^{a_{1}}\right] \leq 6 D_{1}(F) \quad \text { or } \quad \mathbb{E}\left[f^{a_{1}}\right] \geq 1-6 D_{1}(F),
$$

and similarly for $g^{a_{2}}$ and $h^{a_{3}}$.

The rest of the proof is divided into two cases:

- Case A: For all $\sigma \in S_{3}$ we have $D_{1}\left(F^{\sigma}\right) \leq D_{1}(F) / 4$.

- Case B: There exists $\sigma_{0} \in S_{3}$ such that $\overline{D_{1}}\left(F^{\sigma_{0}}\right)>D_{1}(F) / 4$. 
We first show that Case A leads to a contradiction by constructing a GSWF $G^{\prime} \in \mathcal{F}_{3}(n)$ such that $\operatorname{Pr}\left[F \neq G^{\prime}\right]<D_{1}(F)$. Then we show that in Case $\mathrm{B}$, the assertion of the theorem follows by applying Lemma 3.2 to the function $F^{\sigma_{0}}$.

Case A: Consider a GSWF $G^{\prime}$ whose choice functions $f^{\prime \prime}, g^{\prime \prime}, h^{\prime \prime}$ are defined as follows: For $a_{1} \in\{0,1\}$,

$$
f^{\prime \prime}\left(a_{1}, x_{2}, \ldots, x_{n}\right)= \begin{cases}1 \text { (constant), } & \mathbb{E}\left[f^{a_{1}}\right] \geq 1-D_{1}(F) / 4, \\ 0 \text { (constant), } & \mathbb{E}\left[f^{a_{1}}\right] \leq D_{1}(F) / 4, \\ f^{a_{1}}, & \text { otherwise }\end{cases}
$$

and similarly for $g^{\prime \prime}$ and $h^{\prime \prime}$. We claim that the output of $G^{\prime}$ is always transitive, and thus $G^{\prime} \in \mathcal{F}_{3}(n)$. Indeed, by assumption, for any $\sigma \in S_{3}$, there exists $\bar{G}^{\sigma} \in \mathcal{F}_{3}(n-1)$ such that $\operatorname{Pr}\left[F^{\sigma} \neq \bar{G}^{\sigma}\right] \leq D_{1}(F) / 4$. The GSWF $\bar{G}^{\sigma}$ cannot be a dictatorship since by (30), the choice functions $f^{a_{1}}, g^{a_{2}}, h^{a_{3}}$ of $F^{\sigma}$ satisfy

$$
\mathbb{E}\left[f^{a_{1}}\right] \leq 6 D_{1}(F) \quad \text { or } \quad \mathbb{E}\left[f^{a_{1}}\right] \geq 1-6 D_{1}(F),
$$

and thus, for any dictatorship $H$,

$$
\operatorname{Pr}\left[F^{\sigma} \neq H\right] \geq 1 / 2-6 D_{1}(F)>1 / 2-2^{500000} .
$$

Therefore, $\bar{G}^{\sigma}$ always ranks one alternative at the top/bottom. Denote the choice functions of $\bar{G}^{\sigma}$ by $\tilde{f}, \tilde{g}, \tilde{h}$, and assume without loss of generality that $\bar{G}^{\sigma}$ always ranks alternative 1 at the top, and thus $\tilde{f}=1$ and $\tilde{h}=0$. Since

$$
D_{1}(F) / 4 \geq \operatorname{Pr}\left[F^{\sigma} \neq \bar{G}^{\sigma}\right] \geq \max \left(\operatorname{Pr}\left[f^{a_{1}} \neq \tilde{f}\right], \operatorname{Pr}\left[g^{a_{2}} \neq \tilde{g}\right], \operatorname{Pr}\left[h^{a_{3}} \neq \tilde{h}\right]\right),
$$

it follows that

$$
\mathbb{E}\left[f^{a_{1}}\right] \geq 1-D_{1}(F) / 4 \quad \text { and } \quad \mathbb{E}\left[h^{a_{3}}\right] \leq D_{1}(F) / 4 .
$$

Hence, by the definition of $G^{\prime}$, its choice functions satisfy $f^{\prime \prime}=1$ and $h^{\prime \prime}=0$, which means that $G^{\prime}$ always ranks alternative 1 at the top, and is thus always transitive.

Therefore, $G^{\prime} \in \mathcal{F}_{3}(n)$, and on the other hand, we have

$$
\operatorname{Pr}\left[F \neq G^{\prime}\right] \leq \operatorname{Pr}\left[f \neq f^{\prime \prime}\right]+\operatorname{Pr}\left[g \neq g^{\prime \prime}\right]+\operatorname{Pr}\left[h \neq h^{\prime \prime}\right] \leq 3 \cdot D_{1}(F) / 4<D_{1}(F),
$$

contradicting the definition of $D_{1}(F)$.

Case B: Let $\sigma_{0} \in S_{3}$ be such that $D_{1}\left(F^{\sigma_{0}}\right)>D_{1}(F) / 4$. By (30), the choice functions $f^{a_{1}}, g^{a_{2}}, h^{a_{3}}$ of $F^{\sigma_{0}}$ satisfy

$$
\mathbb{E}\left[f^{a_{1}}\right] \leq 6 D_{1}(F) \quad \text { or } \quad \mathbb{E}\left[f^{a_{1}}\right] \geq 1-6 D_{1}(F),
$$

and thus (in the notation of Lemma 3.2) $D_{2}^{\prime}\left(F^{\sigma_{0}}\right) \leq 6 D_{1}(F)<2^{-500000}$. Hence, we can apply Lemma 3.2 to the GSWF $G^{\sigma_{0}}$, and get

$$
P\left(F^{\sigma_{0}}\right) \geq \frac{1}{10} \cdot \operatorname{RHC}\left(D_{1}\left(F^{\sigma_{0}}\right) / 2, D_{1}\left(F^{\sigma_{0}}\right) / 2\right) \geq \frac{1}{10}\left(D_{1}(F) / 8\right)^{3}=\frac{1}{5120} \cdot D_{1}(F)^{3} .
$$


Finally,

$$
P(F)=\frac{1}{6} \sum_{\sigma \in S_{3}} P\left(F^{\sigma}\right) \geq \frac{1}{6} \cdot P\left(F^{\sigma_{0}}\right)>\frac{1}{50000} D_{1}(F)^{3} .
$$

This completes the proof of the theorem.

Theorem 1.3 follows immediately from Theorem 4.1 using Theorem 2.14 (the generic reduction lemma of Mossel).

\subsection{Proof of Theorem 1.5}

Theorem 4.2. There exists an absolute constant $C$ such that for any GSWF $F$ on three alternatives that satisfies the IIA condition, if the probability of non-transitive outcome in $F$ is at most

$$
\delta(\epsilon)=\min \left(C, \frac{1}{10000} \cdot \epsilon^{\frac{9\left(\sqrt{\log _{2}(1 / \epsilon)}+1 / 3\right)^{2}}{8 \log _{2}(1 / \epsilon)}}\right),
$$

then $D_{2}(F) \leq \epsilon$.

Proof. By (8), it is sufficient to prove that for any $\epsilon>0$, if $D_{2}(F)=\epsilon$, then

$$
P(F) \geq \min \left(C, \frac{1}{5000} \cdot \operatorname{RHC}(1 / 2, \epsilon)\right),
$$

for a universal constant $C$. We shall prove this for

$$
C=\exp \left(-\frac{C^{\prime}}{\left(2^{-500003}\right)^{21}}\right)
$$

where $C^{\prime}$ is the constant in Mossel's Theorem 2.13. Let $F$ be a GSWF on three alternatives satisfying the IIA conditions, and denote the choice functions of $F$ by $f, g, h$, as in the proof of Lemma 3.2.

First we consider the case $D_{2}(F) \geq 2^{-500003}$. We show that in general, $D_{1}(F) \geq$ $D_{2}(F)$, and thus in this case we have $D_{1}(F) \geq D_{2}(F) \geq 2^{-500003}$, which by Theorem 2.13 implies that $P(F) \geq C$. Let $G \in \mathcal{F}_{3}(n)$ satisfy $\operatorname{Pr}[F \neq G]=D_{1}(F)$, and denote the Boolean choice functions of $G$ by $f^{\prime}, g^{\prime}, h^{\prime}$. Clearly,

$$
D_{1}(F)=\operatorname{Pr}[F \neq G] \geq \max \left(\operatorname{Pr}\left[f \neq f^{\prime}\right], \operatorname{Pr}\left[g \neq g^{\prime}\right], \operatorname{Pr}\left[h \neq h^{\prime}\right]\right) .
$$

By Theorem 2.15, $G$ either always ranks one alternative at the top/bottom or is a dictatorship. In the first case, at least two of the functions $f^{\prime}, g^{\prime}, h^{\prime}$ are constant, and thus (31) implies that at least two of the functions $f, g, h$ are at most $D_{1}(F)$ away from a constant function. In the latter case, the functions $f^{\prime}, g^{\prime}, h^{\prime}$ are dictatorships, and thus (31) implies that $f, g$, and $h$ are at most $D_{1}(F)$ away from a dictatorship. Hence, in both cases,

$$
D_{2}(F)=\min _{1 \leq i<j \leq 3} \min _{G \in \mathcal{G}_{2}(n)} \operatorname{Pr}\left[F_{i j} \neq G\right] \leq D_{1}(F),
$$

as asserted. 
Now we consider the case $D_{2}(F)<2^{-500003}$. Assume without loss of generality that the minimal distance $\min _{G \in \mathcal{G}_{2}(n)} \operatorname{Pr}\left[F_{i j} \neq G\right]$ is obtained by the choice function $f$, and let $\tilde{f} \in \mathcal{G}_{2}(n)$ satisfy $\operatorname{Pr}[f \neq \tilde{f}]=D_{2}(F)$. If $\tilde{f}$ is a constant function, then in the notation of Lemma 3.2, this implies that $D_{2}^{\prime}(F)=D_{2}(F)<2^{-500003}$, and thus we can apply Lemma 3.2 to $F$ and get

$$
P(F) \geq \frac{1}{10} \cdot \operatorname{RHC}\left(1 / 2, D_{2}^{\prime}(F)\right)>\frac{1}{5000} \cdot \operatorname{RHC}\left(1 / 2, D_{2}(F)\right),
$$

as asserted. Thus, we may assume that $\tilde{f}$ is a dictatorship.

Assume that $\tilde{f}$ is a dictatorship of the first voter. Define the functions $F^{\sigma}, f^{0}, f^{1}, g^{0}$, $g^{1}, h^{0}, h^{1}$ as in the proof of Theorem 4.1, and let

$$
\tilde{f}^{0}\left(x_{2}, \ldots, x_{n}\right)=\tilde{f}\left(0, x_{2}, \ldots, x_{n}\right) \text { and } \tilde{f}^{1}\left(x_{2}, \ldots, x_{n}\right)=\tilde{f}\left(1, x_{2}, \ldots, x_{n}\right) .
$$

Clearly, we have

$$
D_{2}(F)=\operatorname{Pr}[f \neq \tilde{f}]=\frac{1}{2}\left(\operatorname{Pr}\left[f^{0} \neq \tilde{f}^{0}\right]+\operatorname{Pr}\left[f^{1} \neq \tilde{f}^{1}\right]\right),
$$

and thus, for $a_{1} \in\{0,1\}$,

$$
\operatorname{Pr}\left[f^{a_{1}} \neq \tilde{f}^{a_{1}}\right] \leq 2 D_{2}(F) .
$$

Since $\tilde{f}^{0}$ and $\tilde{f}^{1}$ are constant functions, this implies that

$$
\mathbb{E}\left[f^{a_{1}}\right] \leq 2 D_{2}(F) \quad \text { or } \quad \mathbb{E}\left[f^{a_{1}}\right] \geq 1-2 D_{2}(F) .
$$

The rest of the proof is divided into two cases:

- Case A: For all $\sigma \in S_{3}$ we have $D_{2}^{\prime}\left(F^{\sigma}\right) \leq D_{2}(F) / 4$.

- Case B: There exists $\sigma_{0} \in S_{3}$ such that $D_{2}^{\prime}\left(F^{\sigma_{0}}\right)>D_{2}(F) / 4$.

Case A: In this case, for any $\sigma \in S_{3}$, at least one of the choice functions of $F^{\sigma}$ is at most $D_{2}(F) / 4$ away from a constant function. Note that if $f^{0}$ is at most $D_{2}(F) / 4$ away from a constant function, then $f^{1}$ must be at least $7 D_{2}(f) / 4$ away from a constant function, since otherwise $f$ is less than $D_{2}(F)$ away either from a constant function or from a dictatorship, contradicting the definition of $D_{2}(F)$. The same holds also for the pairs $\left(g^{0}, g^{1}\right)$ and $\left(h^{0}, h^{1}\right)$. Thus, the only two possibilities are that either the functions $f^{1}, g^{1}, h^{1}$ or $f^{0}, g^{0}, h^{0}$ are simultaneously at most $D_{2}(F) / 4$ away from a constant function. (For example, if $f^{1}, g^{1}, h^{0}$ are at most $D_{2}(F) / 4$ away from a constant function, then $f^{0}, g^{0}, h^{1}$ are at least $7 D_{2}(F) / 4$ away from a constant function, and thus, for the preference $\sigma=(0,0,1)$, we have $D_{2}^{\prime}\left(F^{\sigma}\right) \geq 7 D_{2}(F) / 4$, a contradiction. The other possibilities are discarded in a similar way.) Assume that $f^{1}, g^{1}, h^{1}$ are at most $D_{2}(F) / 4$ away from a constant function. Furthermore, since amongst the expectations $\mathbb{E}\left[f^{1}\right], \mathbb{E}\left[g^{1}\right], \mathbb{E}\left[h^{1}\right]$, at least two are close to 1 or at least two are close to zero, we can assume that

$$
\operatorname{Pr}\left[f^{1} \neq 1\right] \leq D_{2}(F) / 4 \text { and } \operatorname{Pr}\left[g^{1} \neq 1\right] \leq D_{2}(F) / 4 .
$$


Consider the GSWF $F^{\sigma_{0}}$ for the preference $\sigma_{0}=(1,1,0)$. Since $h^{0}$ is at least $7 D_{2}(F) / 4$ away from the constant zero function, it follows that

$$
\begin{aligned}
P\left(F^{\sigma}\right) & \geq \operatorname{Pr}_{\text {profile } \in\left(S_{3}\right)^{n-1}}\left[\left(f^{1}, g^{1}, h^{0}\right)(\text { profile })=(1,1,1)\right] \\
& \geq 7 D_{2}(F) / 4-D_{2}(F) / 4-D_{2}(F) / 4=5 D_{2}(F) / 4,
\end{aligned}
$$

and thus

$$
P(F)=\frac{1}{6} \sum_{\sigma \in S_{3}} P\left(F^{\sigma}\right) \geq \frac{1}{6} \cdot P\left(F^{\sigma_{0}}\right) \geq \frac{5}{24} \cdot D_{2}(F)>\frac{1}{5000} \cdot \operatorname{RHC}\left(1 / 2, D_{2}(F)\right),
$$

as asserted.

Case B: Let $\sigma_{0} \in S_{3}$ be such that $D_{2}^{\prime}\left(F^{\sigma_{0}}\right)>D_{2}(F) / 4$. By (32), the choice function $f^{a_{1}}$ of $F^{\sigma_{0}}$ satisfies

$$
\mathbb{E}\left[f^{a_{1}}\right] \leq 2 D_{2}(F) \quad \text { or } \quad \mathbb{E}\left[f^{a_{1}}\right] \geq 1-2 D_{2}(F),
$$

and thus $D_{2}^{\prime}\left(F^{\sigma_{0}}\right) \leq 2 D_{2}(F)<2^{-500000}$. Hence, we can apply Lemma 3.2 to the GSWF $G^{\sigma_{0}}$, and get

$$
\begin{aligned}
P\left(F^{\sigma_{0}}\right) & \geq \frac{1}{10} \cdot \operatorname{RHC}\left(1 / 2, D_{2}^{\prime}\left(F^{\sigma_{0}}\right)\right) \geq \frac{1}{10} \cdot \operatorname{RHC}\left(1 / 2, D_{2}(F) / 4\right) \\
& \geq \frac{1}{640} \cdot \operatorname{RHC}\left(1 / 2, D_{2}(F)\right) .
\end{aligned}
$$

Finally,

$$
P(F)=\frac{1}{6} \sum_{\sigma \in S_{3}} P\left(F^{\sigma}\right) \geq \frac{1}{6} \cdot P\left(F^{\sigma_{0}}\right)>\frac{1}{5000} \cdot \operatorname{RHC}\left(1 / 2, D_{2}(F)\right) .
$$

This completes the proof of the theorem.

The generalization to $k$ alternatives for all $k \geq 3$ follows immediately by applying Theorem 4.2 to any subset of three alternatives.

\section{Tightness of results}

In this section we show that for GSWFs on three alternatives, the assertions of Theorems 1.3 and 1.5 are tight up to logarithmic factors. In all our examples below, the Boolean choice functions $f, g, h$ of the GSWF $F$ are monotone threshold functions, that is, functions of the form

$$
(f(x)=1) \Leftrightarrow\left(\sum_{i=1}^{n} x_{i} \geq l\right)
$$


for different values of $l$. We note that in [20, Theorem 2.9], Mossel et al. showed that amongst neutral GSWFs on three alternatives, a GSWF based on the majority rule is the "most rational" in the asymptotic sense (i.e., has the least probability of non-transitive outcome as the number of voters tends to infinity). To some extent, our examples generalize this result to general GSWFs on three alternatives. The examples show that GSWFs based on monotone threshold Boolean choice functions are "close to most rational" amongst GSWFs whose choice functions have the same expectations, in the sense that their probability of non-transitive outcome is logarithmic close to the lower bound. In fact, we conjecture that such GSWFs are indeed the most rational amongst GSWFs whose choice functions have the same expectations. However, such an exact result is not known even for neutral GSWFs.

We use the following proposition of Mossel et al. [19], showing that Borell's reverse Bonami-Beckner inequality is essentially tight for diametrically opposed Hamming balls. Since we use the proposition only for noise of rate $\epsilon=1 / 3$, we state it in this particular case.

Theorem 5.1 ([19, Proposition 3.9]). Fix $s, t>0$, and let $f_{n}, g_{n}:\{0,1\}^{n} \rightarrow\{0,1\}$ be defined by

$\left(f_{n}(x)=1\right) \Leftrightarrow\left(\sum_{i=1}^{n} x_{i} \leq \frac{n}{2}-\frac{s}{2} \sqrt{n}\right) \quad$ and $\quad\left(g_{n}(x)=1\right) \Leftrightarrow\left(\sum_{i=1}^{n} x_{i} \geq \frac{n}{2}+\frac{t}{2} \sqrt{n}\right)$.

Then

$$
\lim _{n \rightarrow \infty} \sum_{S \subset\{1, \ldots, n\}}\left(\frac{1}{3}\right)^{|S|} \hat{f}_{n}(S) \hat{g}_{n}(S) \leq \frac{\sqrt{8 / 9}}{2 \pi s(s / 3+t)} \exp \left(-\frac{1}{2} \frac{s^{2}+2 s t / 3+t^{2}}{8 / 9}\right) .
$$

In order to show the tightness of Theorem 1.3, we fix a constant $\epsilon>0$ and define the choice functions according to (33), choosing the values of $l$ such that

$$
\mathbb{E}[f]=0, \quad \mathbb{E}[g]=1-\epsilon, \quad \mathbb{E}[h]=1-\epsilon .
$$

It is clear that $D_{1}(F)=\epsilon$. By $(22)$,

$$
P(F)=\left\langle T_{1 / 3}(1-g), \bar{h}\right\rangle .
$$

By our construction, the pair of functions $(1-g, \bar{h})$ is of the form considered in Theorem 5.1 with $s=t \approx \sqrt{2 \log (1 / \epsilon)}$, and thus by the theorem, for $n$ sufficiently large,

$P(F)=\left\langle T_{1 / 3}(1-g), \bar{h}\right\rangle \leq \frac{\sqrt{8 / 9}}{2 \pi s(s / 3+t)} \exp \left(-\frac{1}{2} \frac{s^{2}+2 s t / 3+t^{2}}{8 / 9}\right) \approx C \epsilon^{3} \log (1 / \epsilon)$.

The lower bound asserted by Theorem 1.3 is $P(F) \geq C^{\prime} \epsilon^{3}$, and thus the example shows the tightness of the assertion up to logarithmic factors.

The tightness of Theorem 1.5 is shown similarly, with choice functions chosen so that

$$
\mathbb{E}[f]=\epsilon, \quad \mathbb{E}[g]=1-\epsilon, \quad \mathbb{E}[h]=1 / 2 .
$$


It is clear that $D_{2}(F)=\epsilon$, and by (22),

$$
P(F) \leq\left\langle T_{1 / 3} f^{\prime}, h\right\rangle+\left\langle T_{1 / 3}(1-g), \bar{h}\right\rangle .
$$

The pairs $\left(f^{\prime}, h\right)$ and $(1-g, \bar{h})$ are each of the form considered in Theorem 5.1, and application of the theorem to both yields tightness up to a logarithmic factor, as in the previous case.

Finally, we note that while the examples above deal with GSWFs whose choice functions have constant expectation, it also makes sense to consider choice functions whose expectation tends to zero as $n$ (the number of voters) tends to infinity. In particular, one may ask what is the least possible probability of non-transitive outcome, as a function of $n$, for GSWFs with $D_{1}(F)>0$ or $D_{2}(F)>0$. It appears that the question is of interest mainly for $D_{2}(F)$, as for $D_{1}(F)$ one can easily check that the minimal possible probability of $6^{-n}$ is obtained by a GSWF whose choice functions are chosen according to (33) with

$$
\mathbb{E}[f]=0, \quad \mathbb{E}[g]=1-2^{-n}, \quad \mathbb{E}[h]=1-2^{-n} .
$$

For $D_{2}(F)$, it was shown in [17] that for a GSWF whose choice functions are chosen according to (33) with

$$
\mathbb{E}[f]=2^{-n}, \quad \mathbb{E}[g]=1-2^{-n}, \quad \mathbb{E}[h]=1 / 2,
$$

we have $P(F) \leq 0.471^{n}$. Furthermore, it was conjectured that this is the most rational GSWF on three alternatives that satisfies the assumptions of Arrow's theorem (and in particular, the minimal possible probability $6^{-n}$ is not attained). Our results show that this function is at least close to most rational, as by Theorem 1.5, for any GSWF $F$ such that $D_{2}(F)>0$, we have

$$
P(F) \geq C \cdot \operatorname{RHC}\left(1 / 2,2^{-n}\right) \approx C \cdot 0.458^{n} .
$$

\section{Questions for further research}

We conclude this paper with several open problems related to our results.

- Our main lemma (Lemma 3.2) gives an essentially tight lower bound on the probability of non-transitive outcome for GSWFs in which at least one of the Boolean choice functions is close to a constant function. In the case where the distance from constant functions is greater than a fixed constant, our technique fails, and we use Mossel's theorem [18] instead. As a result, the constant multiplicative factor in the assertions of Theorems 1.3 and 1.5 is extremely small, and clearly non-optimal. It will be interesting to find a direct proof also for GSWFs whose Boolean choice functions are far from constant functions, thus removing the reliance of the proof on the non-linear invariance principle (used in Mossel's argument) that seems unnatural in our context, and improving the constant factor. 
- While the results of Kalai [14] and Mossel [18] hold also for more general distributions of the individual preferences called "even product distributions" or "symmetric distributions" (see $[17,18]$ ), our proof does not extend directly to such distributions. The reason is that for highly biased distributions of the preferences, the lower bound obtained by Borell's reverse Bonami-Beckner inequality is weaker, and cannot beat the upper bound obtained by the Bonami-Beckner inequality. Thus, obtaining a tight quantitative version of Arrow's theorem for general even product distributions of the preferences is an interesting open problem.

- We believe that GSWFs whose Boolean choice functions are monotone threshold functions are the most rational amongst GSWFs whose choice functions have the same expectations, not only in the asymptotic sense, but also for any particular (large enough) $n$. However, this conjecture seems quite challenging, as it includes the Majority is Stablest conjecture (whose proof by Mossel et al. [20] holds only in the limit as $n \rightarrow \infty$ ).

- Another direction of research is using our techniques to obtain quantitative versions of other theorems in social choice theory. In [8], Friedgut et al. presented a quantitative version of the Gibbard-Satterthwaite theorem $[11,21]$ for neutral GSWFs on three alternatives. Recently, Isaksson et al. [12] generalized the result of [8] to neutral GSWFs on $k$ alternatives, for all $k \geq 4$. One of the main ingredients in the proof of [8] is Kalai's quantitative Arrow theorem for neutral GSWFs. It seems interesting to find out whether our quantitative version of Arrow's theorem can lead to a quantitative Gibbard-Satterthwaite theorem for general GSWFs (without the neutrality assumption).

- Finally, our results (as well as the previous results of Kalai [14] and Mossel [18]) apply only to GSWFs that satisfy the IIA condition, since such GSWFs can be represented by their Boolean choice functions, which allows using the tools of discrete harmonic analysis. It will be very interesting to find a quantitative version of Arrow's theorem that will not assume the IIA condition, but rather will relate the probability of nontransitive outcome to the distance of the GSWF from satisfying IIA.

Acknowledgments. It is a pleasure to thank Gil Kalai and Elchanan Mossel for numerous fruitful discussions that motivated our work.

The work was done partly while the author was at the Weizmann Institute of Science and was supported by the Koshland Center for Basic Research, and partly while the author was at the Hebrew University of Jerusalem and was supported by the Adams Fellowship Program of the Israeli Academy of Sciences and Humanities.

\section{References}

[1] Arrow, K. J.: A difficulty in the concept of social welfare. J. Political Economy 58, 328-346 (1950)

[2] Barbera, S.: Pivotal voters: A new proof of Arrow's theorem. Economics Lett. 6, 13-16 (1980) MR 0614478

[3] Beckner, W.: Inequalities in Fourier analysis. Ann. of Math. 102, 159-182 (1975) Zbl 0338.42017 MR 0385456

[4] Bonami, A.: Étude des coefficients de Fourier des fonctions de $L^{p}(G)$. Ann. Inst. Fourier (Grenoble) 20, 335-402 (1970) Zbl 0195.42501 MR 0283496 
[5] Borell, C.: Positivity improving operators and hypercontractivity. Math. Z. 180, 225-234 (1982) Zbl 0472.47015 MR 0661699

[6] de Condorcet, M.: An Essay on the Application of Probability Theory to Plurality Decision Making (1785)

[7] Feige, U., Kindler, G., O’Donnell, R.: Understanding parallel repetition requires understanding foams. Electron. Colloq. Comput. Complexity, Report No. 43 (2007)

[8] Friedgut, E., Kalai, G., Nisan, N.: Elections can be manipulated often. In: Proc. 49-th Ann. Sympos. on Foundations of Comp. Sci. (FOCS), 243-249 (2009)

[9] Gehrlein, W. V.: Condorcet's paradox and the Condorcet efficiency of voting rules. Math. Japon. 45, 173-199 (1997) Zbl 0869.90013 MR 1434971

[10] Geneakoplos, J.: Three brief proofs of Arrow's impossibility theorem. Cowels Foundation Discussion Paper number 1123R, Yale Univ. (1997); http://ideas.uqam.ca/ideas/data/Papers /cwlcwldpp1123R.html

[11] Gibbard, A.: Manipulation of voting schemes: a general result. Econometrica 41, 587-601 (1973) Zbl 0325.90081 MR 0441407

[12] Isaksson, M., Kindler, G., Mossel, E.: The geometry of manipulation-A quantitative proof of the Gibbard-Satterthwaite theorem. Combinatorica 32, 221-250 (2012) MR 2927640

[13] Kahn, J., Kalai, G., Linial, N.: The influence of variables on Boolean functions. In: Proc. 29-th Ann. Sympos. on Foundations of Comp. Sci. (FOCS), Computer Soc. Press, 68-80 (1988)

[14] Kalai, G.: A Fourier-theoretic perspective on the Condorcet paradox and Arrow's theorem. Adv. Appl. Math. 29, 412-426 (2002) Zbl 1038.91027 MR 1942631

[15] Kalai, G.: Private communication (2007)

[16] Kalai, G., Safra, M.: Threshold phenomena and influence. In: Computational Complexity and Statistical Physics, A. G. Percus et al. (eds.), Oxford Univ. Press, New York, 25-60 (2006) Zbl 1156.82317 MR 2208732

[17] Keller, N.: On The probability of a rational outcome for generalized social welfare functions on three alternatives. J. Combin. Theory Ser. A 117, 389-410 (2010) Zbl 1189.91055 MR 2592890

[18] Mossel, E.: A quantitative Arrow theorem. Probab. Theory Related Fields, to appear; arXiv:0903.2574v4 (2009)

[19] Mossel, E., O'Donnell, R., Regev, O., Steif, J. E., Sudakov, B.: Non-interactive correlation distillation, inhomogeneous Markov chains, and the reverse Bonami-Beckner inequality. Israel J. Math. 154, 299-336 (2006) Zbl 1140.60007 MR 2254545

[20] Mossel, E., O'Donnell, R., Oleszkiewicz, K.: Noise stability of functions with low influences: invariance and optimality. Ann. of Math. 171, 295-341 (2010) Zbl 1201.60031 MR 2630040

[21] Satterthwaite, M. A.: Strategy-proofness and Arrow's conditions: existence and correspondence theorems for voting procedures and social welfare functions. J. Economic Theory 10, 187-217 (1975) Zbl 0315.90088 MR 0414051

[22] Wilson, R.: Social choice theory without the Pareto principle. J. Economic Theory 5, 478-486 (1972) MR 0449494 